Sabina Hunziker

Laura Laschinger

Simone Portmann-Schwarz

Norbert K. Semmer

Franziska Tschan

Stephan Marsch

\section{Perceived stress and team performance during a simulated resuscitation}

Received: 28 March 2011

Accepted: 30 May 2011

Published online: 22 June 2011

C Copyright jointly held by Springer and ESICM 2011

S. Hunziker, L. Laschinger, and

S. Portmann-Schwarz equally contributed to this work.

Electronic supplementary material

The online version of this article (doi:10.1007/s00134-011-2277-2) contains supplementary material, which is available to authorized users.

\section{S. Hunziker $(\varpi)$}

Department of Medicine,

Division of Pulmonary, Critical Care, and Sleep Medicine, Harvard Medical School, Beth Israel Deaconess Medical Center, Boston, MA, USA

e-mail: HunzikerS@post.harvard.edu

S. Hunziker - L. Laschinger ·

S. Portmann-Schwarz · S. Marsch

Medical Intensive Care Unit,

University Hospital Basel,

Basel, Switzerland

N. K. Semmer

University of Bern, Bern, Switzerland

F. Tschan

University of Neuchâtel, Neuchâtel,

Switzerland
Abstract Purpose: Barriers to optimal performance of cardiopulmonary resuscitation may partly relate to human factors, such as stress and specific emotions. The aim of this study was to investigate whether mental stress and different perceived emotions have a negative impact on the performance of rescuers. Methods: This prospective, observational study was conducted at the Simulator Center of the University Hospital Basel, Switzerland. A total of 120 medical students (70\% female) participated in teams of three. They reported levels of perceived stress, feeling overwhelmed, motivation and specific emotions before, during, and after a simulated resuscitation. The association of stress/overload (index of stress and feeling overwhelmed), motivation, and specific emotions with resuscitation performance defined as hands-on time during the first $180 \mathrm{~s}$ after cardiac arrest was investigated. Results: During resuscitation, levels of stress/overload, motivation, and negative emotions were significantly higher as compared to the periods before and after resuscitation. In contrast, positive emotions were highest before and after resuscitation and significantly lower during resuscitation. In general, females reported higher stress/overload and negative emotions, whereas males reported more positive emotions. A multivariate linear regression model showed negative associations of stress/overload (regression coefficient -18.12 , 95\% CI -30.73 , $-5.51, p=0.006)$ and positive associations of motivation (regression coefficient $13.45,95 \%$ CI $0.95,25.95$, $p=0.036$ ) with resuscitation performance. Conclusion: A simulated cardiac arrest caused substantial perceived stress/overload and negative emotions, particularly in female students, which adversely impacted resuscitation performance. Further studies are required to expand our findings to more experienced medical professionals and investigate whether stress coping strategies improve resuscitation performance.

\section{Keywords Stress - Resuscitation ·} Human factors - Emotion . Emotion wheel - Outcome . Team performance

\section{Introduction}

Cardiopulmonary resuscitation (CPR) following cardiac arrest is the most important determinant of mortality, and survival decreases by about $7-10 \%$ for every minute without CPR $[1,2]$. Different factors may influence the quality of CPR, including the personal experience and technical skills of rescuers. Importantly, other human factors, such as team 
behavior and teambuilding, are also associated with performance and outcomes of CPR [3-9]. Being involved in a medical emergency - such as resuscitation-is a stressful and emotionally demanding experience [10]. It has become clear that acute stress potentially modifies human performance on an individual and a team level [11-13]. For example, stress of individual surgeons was associated with impairment in their surgical performance, leading to adverse patient outcomes [14]. In addition, stress has been shown to cause reduced team perspective, which potentially impacts the management of an emergency situation [15]. Yet, little is known about perceived stress of rescuers during CPR and whether stress is associated with CPR performance. One could assume that stress impairs performance of CPR teams. On the other hand, stress may help rescuers to mobilize energy and "protect" performance in key aspects [16]. These compensatory strategies may prevent the negative effects of stress from unfolding during the relatively short time of a resuscitation, especially when the task is not too demanding [17].

Perceived stress is a summary term that refers to an "aversive feeling state" that can diminish one's well-being, particularly when people realize that the demands placed on them exceed their ability to cope [18]. There are many affective reactions that can lead to such a state and thus cause perceived stress. In an achievement context, feelings of overload are likely to be pervasive $[13,16]$, but emotions such as anxiety, worry, or shame may play a role as well. In addition, the experience of stress often has a challenge component, which implies (a) high motivation, and (b) positive emotions such as pride if success is obtained [19].

The primary aim of this study was to investigate whether mental stress has a negative impact on the performance of rescuers in a simulated cardiac arrest scenario. Because stress refers to different emotional states, we not only assessed perceived stress levels, but also levels of feeling overwhelmed, motivated, and different positive and negative emotions. We hypothesized that (a) rescuers would experience substantial stress with dynamical changes before, during, and after the CPR situation; and (b) that higher stress predicts lower CPR performance in regard to hands-on time. Knowledge of which specific emotions are associated with a stressful encounter (such as a resuscitation) and whether these emotions predict performance over and above general accounts of stress may help to find specific coping strategies to reduce stress in rescuers, potentially leading to better CPR performance.

\section{Methods}

Study design and participants

This prospective, observational study was performed between November 2006 and December 2006 at the
Simulator Center of the University Hospital in Basel, Switzerland. We offered optional workshops to fourth year medical students who did not have any prior teaching about CPR algorithms. Participants were blinded in regard to the hypotheses of the study. Students were randomly allocated to teams of three rescuers.

The study was approved by the local institutional review board (Ethikkommission beider Basel, Switzerland, EKBB). All students gave written informed consent.

\section{Simulator}

The study was performed in a high fidelity simulator setting, which has been demonstrated to create high perceived realism [4-6, 20]. We used a full-body computerbased manikin (Human Patient Simulator, METI, Sarasota, FL, USA) [5, 20].

Scenario and study protocol

All teams performed the same cardiac arrest scenario (see details below, time points 1-11) which persisted for at least $3 \mathrm{~min}$ independent of the team's actions. Then, sinus rhythm could be restored with defibrillation. After the patient woke up, a senior physician joined, asking for a detailed handover followed by a video-assisted debriefing. Upon completion of the scenario, participants filled out questionnaires that measured 18 discrete emotions using the Geneva emotion wheel [21]. For each time point, students also reported their level of feeling stressed, motivated, and overwhelmed on a Likert scale from 1 to 10 ( 1 being lowest and 10 being highest). The following time points were included (see legend of Fig. 1): (1) students receive instructions about the patient's history in front of the simulator room, (2) team enters the simulator room, (3) students start taking the patient's history, (4) occurrence of the cardiac arrest, (5) decision-making within the team about CPR, (6) time until the first defibrillation, (7) moment of first defibrillation, (8) time after defibrillation, later period of CPR, (9) manikin awakes, (10) senior physician enters the room and students have to give report. After the debriefing (11), the students answered the same questions with regard to how they felt during the debriefing. We defined the following time periods: before resuscitation period (1-3), early resuscitation period (4-6), late resuscitation period (6-8), after resuscitation period (9-11).

\section{Emotion wheel}

The emotion wheel was developed for verbally reporting emotions in a way that is easy to handle and allows quantification of the intensity of a variety of emotions 

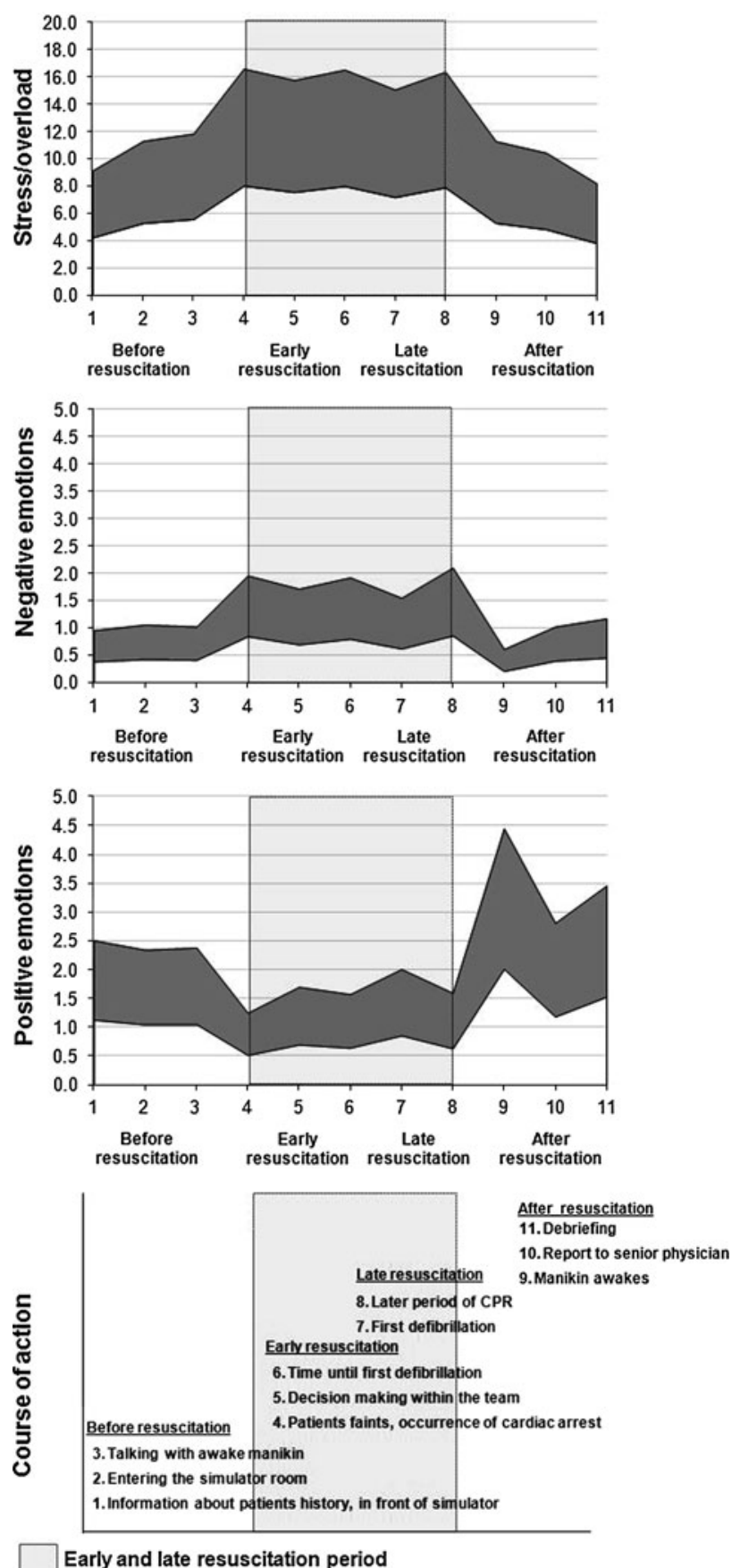

Fig. 1 Stress/overload index, positive emotions, and negative emotions during different time points before, during, and after resuscitation; Figure shows upper and lower 95\% confidence limit at the 11 different time points

[21]. The version we used contains 18 emotions, which were assessed on a Likert scale from 0 to 5 (0, this emotion was not felt; 5, this emotion was felt most strongly). Not all of the emotions are relevant in our context, which is achievement-related. We defined negative emotions as irritation, disappointment, guilt, shame, anxiety, and desperation, and positive emotions as interest, pride, joy, pleasure, and relief.

\section{Outcomes and their measurement}

To evaluate the quality of performance of the simulated resuscitation on a team level, we used the amount of hands-on time, defined as duration of uninterrupted chest compressions or defibrillation in the first $180 \mathrm{~s}$ after the onset of the cardiac arrest, as our endpoint [4]. Each defibrillation was regarded as $10 \mathrm{~s}$ of hands-on time. Interruptions of chest compressions to perform ventilation were rated as continuous hands-on time if the interruption was less than $10 \mathrm{~s}$. Based on the video tapes of the simulation, performance was coded, second by second.

\section{Statistical analysis}

We analyzed perceived stress, motivation, and emotions both on the individual and group level. For the analyses on the individual level, continuous variables are shown in Fig. 1 as upper and lower $95 \%$ confidence intervals at the different time points. Intra-individual differences across time periods were analyzed using within-subject analysis of variance (WS-ANOVA) and paired $t$ tests; gender differences were analyzed by $t$ tests for independent groups. As hands-on time is a group-level outcome, group data were used to analyze the associations of stress, motivation, and emotions with performance; for these analyses the data were aggregated across individual team members. For analyzing associations with outcome, we performed linear regression analyses. To predict hands-on time, we used parameters from the period before resuscitation and from the early resuscitation period. We expected that positive emotions would not predict performance; rather, performance should predict the emergence of positive emotions, most notably pride, which therefore should refer to the period after resuscitation.

As our central interest refers to stress, we first determined if stress was best represented by the single item on "feeling stressed" or by a combination of this item with other specific emotions. Being in an achievement context, with a task that is quite demanding for medical students, we expected the stress item to correlate highly with feeling overwhelmed. This was the case $(r=0.68$, $p<0.01$, for the pre-resuscitation period; $r=0.75$, $p<0.01$, for the early resuscitation period); the two items were therefore combined into a "stress/overload" index. Three specific emotions were correlated with that index (desperate, $r=0.42, p<0.01$; anxious, $r=0.58$, $p<0.01$; ashamed, $r=0.53, p<0.01$ ). Adding them to the index in no case improved its reliability (Cronbach's alpha) but rather decreased it; the final stress/overload 
index therefore consists of the variables stressed and overwhelmed only.

$p$ values less than 0.05 are considered to indicate statistical significance; all tests are two-tailed. Analyses were performed using SPSS/PC (Version 19, SPSS Inc, Chicago, IL, USA) and STATA 9.2 (Stata Corp, College Station, TX).

\section{Results}

Study population

Participants were 120 medical students (70\% female), with a median age of 23 years (IQR 22-24) assigned to 40 teams of three; 14 teams $(35 \%)$ were composed of 2 males and 1 female, 8 teams (20\%) of 2 females and 1 male; and 18 teams (45\%) were all female. CPR was started after a median time of $41 \mathrm{~s}$ (IQR 33-62), and all resuscitation teams started CPR within the first $180 \mathrm{~s}$. The median hands-on time was $43 \mathrm{~s}$ (IQR 21-75) (Table 1).

Perceived stress, motivation, and positive/negative emotions during simulation

Figure 1 shows upper and lower 95\% confidence limits for the stress/overload index, positive emotions, and negative emotions at the different time points. The stress/ overload index showed a similar pattern as negative emotions; they were both lower before resuscitation, increased during resuscitation, and decreased again after resuscitation. Conversely, positive emotions were higher before and after resuscitation and lower in the resuscitation period.

We found significant within-subject differences of stress/overload at the different time points (WS-ANOVA $<0.001$ ). Stress/overload levels were 5.4 (SD 1.6) before resuscitation and significantly increased to 8.2 (SD 1.3) $(p<0.001)$ during the early phase of the resuscitation,

Table 1 Baseline characteristics of resuscitation teams

\begin{tabular}{ll}
\hline Parameter & Value \\
\hline $\begin{array}{l}\text { Number of teams (each with } 3 \text { students) } \\
\text { Gender within resuscitation teams }\end{array}$ & 40 \\
2 males and 1 female & $14(35 \%)$ \\
1 male and 2 females & $8(20 \%)$ \\
3 females & $18(45 \%)$ \\
CPR performance & 43 (IQR 21-75) \\
Hands-on time within first 180 s (s) & 41 (IQR 33-62) \\
Time to start CPR (s) & 119 (IQR 65-155) \\
Time to defibrillation (s) & 66 (IQR 50-101) \\
Time to ventilation (s) &
\end{tabular}

then decreased to 7.8 (SD 1.5) during the late resuscitation period $(p=0.002)$, and then fell to 2.8 (SD 1.4) after resuscitation $(p<0.001)$. Similarly, negative and positive emotions showed significant variation within subjects at the different time points (WS-ANOVA $<0.001$ and $<0.001$, respectively). Negative emotions increased significantly from before to the early resuscitation period, were similar in the early and late resuscitation period, and decreased after resuscitation. Positive emotions were highest before resuscitation, significantly decreased during early and late resuscitation, and again increased after resuscitation.

Gender differences of perceived stress and emotions during simulation

We assessed whether female and male students would perceive stress/overload and specific emotions differently at the four different resuscitation periods (see Table 2 and Appendix). Concerning overall stress/overload, female students reported higher perceived levels during each period; the difference was significant before resuscitation and during the early resuscitation phase. The increase (delta) in stress/overload levels, however, was similar in males and females. Females also reported more negative emotions, the differences being significant for the early resuscitation phase and after resuscitation. Male students tended to report more positive emotions, which reached statistical significance only in the late resuscitation period $(p<0.05)$. There were no gender differences with regard to motivation (data not shown).

Table 2 Gender differences in stress/overload, negative and positive emotions

\begin{tabular}{|c|c|c|c|}
\hline & Male & Female & $p$ \\
\hline \multicolumn{4}{|c|}{ Stress/overload (1-10), mean (SD) } \\
\hline Before resuscitation & $4.90(1.43)$ & $5.69(1.55)$ & 0.01 \\
\hline Early resuscitation & $7.65(1.31)$ & 8.39 (1.19) & 0.005 \\
\hline Late resuscitation & $7.48(1.41)$ & $8.00(1.51)$ & 0.082 \\
\hline After resuscitation & $4.66(1.39)$ & $5.16(1.39)$ & 0.078 \\
\hline \multicolumn{4}{|c|}{ Negative emotions $(0-5)$, mean (SD) } \\
\hline Before resuscitation & $0.45(0.40)$ & $0.54(0.42)$ & 0.283 \\
\hline Early resuscitation & $0.74(0.59)$ & $1.02(0.71)$ & 0.043 \\
\hline Late resuscitation & $0.79(0.89)$ & $0.93(0.75)$ & 0.37 \\
\hline After resuscitation & $0.32(0.38)$ & $0.52(0.50)$ & 0.019 \\
\hline \multicolumn{4}{|c|}{ Positive emotions $(0-5)$, mean (SD) } \\
\hline Before resuscitation & $1.17(0.47)$ & $1.23(0.61)$ & 0.6 \\
\hline Early resuscitation & $0.88(0.72)$ & $0.70(0.55)$ & 0.136 \\
\hline Late resuscitation & $1.21(0.86)$ & $0.80(0.62)$ & 0.014 \\
\hline After resuscitation & $2.05(1.17)$ & $1.66(0.83)$ & 0.075 \\
\hline
\end{tabular}

$S D$ standard deviation; positive emotions mean of interest, pride, joy, pleasure, relief; negative emotions mean of desperation, anxiety, shame, guilt, disappointment, and irritation

Bold values indicate $p<0.05$ 
Association of stress and emotions with CPR performance

In a second step, we analyzed associations of stress and emotions with team performance on a group level in a linear regression model. We expected stress/overload to be associated with lower performance, and motivation with higher performance. We tested a model with these two predictors, taken from the early resuscitation period. Both, stress/overload and motivation predicted performance as expected. Adding stress/overload and motivation from the period before resuscitation did not change the results significantly. We then tested if the model could be further improved by adding specific emotions. Neither the index of all negative emotions nor the index of all positive emotions was significantly associated with performance, and that was true for both the period before resuscitation and the early resuscitation phase. Of the specific emotions, only desperation from the early resuscitation period was significantly associated with performance (univariate analysis: $b=-19.2 ; p=0.044)$. When desperation was added to the model, however, it was not significant, whereas both stress/overload and motivation remained significant. Concerning gender composition within resuscitation groups, there was no significant association with performance; thus gender composition was not further considered for the multivariate model.

The final model therefore remained unchanged as shown in Table 3. The unstandardized regression coefficient of -18.1 for stress/overload implies that moving up the stress/overload index, which ranges from 1 to 10 , by one point corresponds to an average of $18 \mathrm{~s}$ less hand-on time in the first $180 \mathrm{~s}$. The coefficient of $b=13.4$ for motivation implies that moving up on the motivation variable by one point corresponds to about $13 \mathrm{~s}$ more hands-on time. Note that adjusting the final model for gender composition did not show a significant effect of gender and no significant change for stress/overload and motivation was observed.

Table 3 Multivariate model of stress and emotions during resuscitation for prediction of hands-on time

\begin{tabular}{lccccc}
\hline & $\begin{array}{l}\text { Regression } \\
\text { coefficient }\end{array}$ & SE & $p$ & $\begin{array}{l}\text { Lower } \\
95 \% \text { CI }\end{array}$ & $\begin{array}{l}\text { Upper } \\
95 \% \text { CI }\end{array}$ \\
\hline $\begin{array}{l}\text { Stress/overload early } \\
\begin{array}{c}\text { resuscitation } \\
\text { Motivation early } \\
\text { resuscitation }\end{array}\end{array}$ & -18.12 & 6.2 .2 & $\mathbf{0 . 0 0 6}$ & -30.73 & -5.51 \\
\hline
\end{tabular}

Note that (unstandardized) regression coefficients represent the increase in the dependent variable per unit increase in the predictors. Stress/overload represents the average of two items: feeling stressed and feeling overwhelmed

Bold values indicate $p<0.05$
Association of CPR performance with later pride

Pride is an emotion that is based on the experience of success [22]. We therefore expected that performance would be associated with pride at a later time, that is, after resuscitation. As expected, hands-on time was significantly associated with pride (regression coefficient per $60 \mathrm{~s}$ of hands on time 0.6 (9\% CI 0.16-1.03), $p=0.009$ ) in the after resuscitation period. None of the other positive emotions, nor the aggregate of all positive emotions from the period after resuscitation, was associated with performance.

\section{Discussion}

Within this simulator study we investigated the dynamic of perceived levels of stress/overload, motivation, and distinct emotions of students at different time points before, during, and after a simulated resuscitation, and their association with CPR performance. We found that on an individual level, the simulated medical emergency situation caused substantial mental stress and negative emotions during the phase of uncertainty and need for immediate action, easing off and being replaced with positive emotions after successfully handling the event. Females tended to perceive more stress/overload and negative emotions, whereas males tended to perceive more positive emotions; however, there were no significant performance differences between teams with different gender composition. Importantly, we found that on a team level, stress/overload during the early resuscitation period was significantly associated with lower CPR performance.

We found a remarkable increase in reported stress/ overload during the simulated cardiac arrest, followed by a significant decrease after the scenario. These results are in line with a recent study that investigated the effects of two types of simulator training for intensive care professionals [23]. Their results revealed significant increases in biochemical stress markers, namely salivatory cortisol and alpha-amylase, from pre-scenario to immediate postscenario measures, corresponding to the self-report-based results of our study. Interestingly, they found lower salivatory alpha-amylase levels in a subsequent simulation which presumably relates to a "training effect". It would be interesting to assess whether repeated CPR training results in lower perceived stress/overload levels with better CPR performance in our study population.

Psychological stress refers to the negative cognitive and emotional states elicited when people realize that the demands placed on them by the environment exceed their ability to cope $[18,24]$. In our study, it was the appraisal of the situation as overwhelming that defined the stress experience and we thus combined feeling overwhelmed 
and stress in a stress/overload index. Specific emotions did not further improve the stress/overload index, nor did they improve the prediction of performance. Of the positive emotions, only pride after the resuscitation period was associated with performance. As pride is an emotion that results from achievement, this result was expected; it implies that the teams' evaluation of their performance was not unrealistic. This realism cannot be attributed simply to the senior physician's feedback: Additional analyses confirmed that the effects were robust when we considered only pride at time points before debriefing/ feedback.

With regard to gender, we found differences within our study in terms of perception of stress/overload, and positive and negative emotions. Whereas female students tended to report higher levels of stress/overload and negative emotions, male students tended to report more positive emotions. Interestingly, team composition in terms of gender was not significantly associated with performance in this study. Yet, previous research showed that on an individual level, female students had less leadership behavior and had less hands-on time compared to males students [25]. Thus, the impact of gender composition within teams needs further investigation.

Some limitations of this study need consideration. This study assessed stress levels of a small but welldefined cohort of volunteers during a simulated resuscitation by self-report. Furthermore, the self-reports were retrospective. We therefore cannot rule out that participants' memory about the emotions felt during early periods were biased by their knowledge of what happened later. On the other hand, such a bias would likely have produced a greater match between stress, overload, and negative emotions than we found. Nevertheless, assessing biochemical parameters might have provided additional information. Note that previous studies using a similar setting found a close association of self-report data and the biochemical stress markers [23]. We used a high- fidelity simulator with previously documented high perceived realism, which has the advantage of producing similar study conditions for all teams. However, stress levels and perceived emotions during a real-life CPR situation may still be different. This study concentrated on medical students and the results may not unconditionally apply to more experienced physicians and resuscitation teams. However, because inexperienced rescuers are often involved early in situations of cardiac arrest, their training is of outmost importance [26, 27].

\section{Conclusions}

Despite the simulation context and the short duration of the scenario, medical students felt stressed and overwhelmed to a considerable extent, and performed less well when experiencing more stress/overload during the early resuscitation. Further studies are required to replicate these results, to investigate if they apply to experienced medical professionals as well, to investigate gender effects, and to elucidate the role of specific emotions in a context where stress is mainly characterized by qualitative overload. In addition, future studies should address whether increasing the awareness about negative effects of stress/overload improves CPR team performance or possibly further enhances perceived stress and thus decreases performance. Furthermore, the effects of specific stress coping strategies on CPR performance should be evaluated.

Acknowledgments $\mathrm{SH}$ was supported partly by an unrestricted research grant from the Swiss National Foundation (SNF PBBSP3128266) and partly by the University of Basel, Switzerland.

Conflict of interest None declared.

\section{References}

1. Ali B, Zafari AM (2007) Narrative review: cardiopulmonary resuscitation and emergency cardiovascular care: review of the current guidelines. Ann Intern Med 147:171-179

2. Larsen MP, Eisenberg MS, Cummins RO, Hallstrom AP (1993) Predicting survival from out-of-hospital cardiac arrest: a graphic model. Ann Emerg Med 22:1652-1658

3. Perkins GD, Hulme J, Bion JF (2002) Peer-led resuscitation training for healthcare students: a randomised controlled study. Intensive Care Med 28:698-700
4. Hunziker S, Tschan F, Semmer NK, Zobrist R, Spychiger M, Breuer M, Hunziker PR, Marsch SC (2009)

Hands-on time during cardiopulmonary resuscitation is affected by the process of teambuilding: a prospective randomised simulator-based trial. BMC Emerg Med 9:3

5. Hunziker S, Buhlmann $C$, Tschan $F$, Balestra G, Legeret C, Schumacher C, Semmer NK, Hunziker P, Marsch S (2010) Brief leadership instructions improve cardiopulmonary resuscitation in a high-fidelity simulation: a randomized controlled trial. Crit Care Med 38:1086-1091
6. Marsch SC, Muller C, Marquardt K, Conrad G, Tschan F, Hunziker PR (2004) Human factors affect the quality of cardiopulmonary resuscitation in simulated cardiac arrests. Resuscitation 60:51-56

7. Tschan F, Semmer NK, Gautschi D, Hunziker P, Spychiger M, Marsch SU (2006) Leading to recovery: group performance and coordinative activities in medical emergency driven groups. Hum Perform 19:277-304 
8. Hunziker S, Johansson A, Tschan F, Semmer NK, Rock L, Howell MD, Marsch S (2011) Teamwork and leadership in cardiopulmonary resuscitation. J Am Coll Cardiol 57(24):2381-2388

9. Hunziker S, Tschan F, Semmer NK, Howell MD, Marsch S (2010) Human factors in resuscitation: lessons learned from simulator studies. J Emerg Trauma Shock 3:389-394

10. Scott G, Mulgrew E, Smith T (2003) Cardiopulmonary resuscitation: attitudes and perceptions of junior doctors. Hosp Med 64:425-428

11. Driskell JE, Salas E (1996) Stress and human performance. Lawrence Erlbaum, Mahwah, pp 1-48

12. Matthews G (2000) Human performance: cognition stress, and individual differences. Psychology Press, Philadelphia, pp 161-176

13. Matthews G, Campbell SE, Falconer S, Joyner LA, Huggins J, Gilliland K, Grier R, Warm JS (2002) Fundamental dimensions of subjective state in performance settings: task engagement, distress, and worry. Emotion 2:257-271

14. Arora S, Sevdalis N, Nestel D, Woloshynowych M, Darzi A, Kneebone R (2010) The impact of stress on surgical performance: a systematic review of the literature. Surgery 147:318-330 330 e311-316
15. Driskell JA, Salas E, Johnston J (1999) Does stress lead to a loss of team perspective? Group Dyn Theory Res Pract 3:291-302

16. Hockey GRJ (1997) Compensatory control in the regulation of human performance under stress and high workload: a cognitive-energetical framework. Biol Psychol 45:73-93

17. Lazarus RS, Eriksen CW (1952) Effects of failure stress upon skilled performance. J Exp Psychol 43:100-105

18. Ng W, Diener E, Aurora R, Harter J (2009) Affluence, feelings of stress, and well-being. Soc Indic Res 94:257-271

19. Folkman S, Moskowitz JT (2000) Positive affect and the other side of coping. Am Psychol 55:647-654

20. Marsch SCU, Tschan F, Semmer N, Spychiger M, Breuer M, Hunziker PR (2005) Unnecessary interruptions of cardiac massage during simulated cardiac arrests. Eur J Anaesthesiol 22:831-833

21. Scherer KR (2005) What are emotions? And how can they be measured? Soc Sci Inf 44:693-727

22. Ellsworth PC, Scherer KR (2003) Appraisal processes in emotions. In: Davidson RJ, Scherer KR, Goldsmith $\mathrm{HH}$ (eds) Handbook of affective sciences. Oxford, Oxford University Press, pp 572-595
23. Muller MP, Hansel M, Fichtner A, Hardt F, Weber S, Kirschbaum C, Ruder S, Walcher F, Koch T, Eich C (2009) Excellence in performance and stress reduction during two different full scale simulator training courses: a pilot study. Resuscitation 80:919-924

24. Lazarus RS (1993) From psychological stress to the emotions-a history of changing outlooks. Annu Rev Psychol 44:1-21

25. Streiff S, Tschan F, Hunziker $S$, Buehlmann C, Semmer NK, Hunziker P, Marsch S (2011) Leadership in medical emergencies depends on gender and personality. Simul Healthc 6:78-83

26. Caffrey SL, Willoughby PJ, Pepe PE, Becker LB (2002) Public use of automated external defibrillators. N Engl J Med 347:1242-1247

27. Schneider L, Sterz F, Haugk M, Eisenburger P, Scheinecker W, Kliegel A, Laggner AN (2004) CPR courses and semi-automatic defibrillators-life saving in cardiac arrest? Resuscitation 63:295-303 